\title{
TREATMENT OF HARDWARE EXPOSURE AFTER SEVERE INFECTIONS IN SPINE SURGERY WITH PEDICLED MUSCULAR FLAPS
}

\author{
Alvaro Baik Cho, Luciano Miller Reis Rodrigues, Rodrigo Junqueira Nicolau, Gustavo Mantovani Rugiero, Walter Yoshinori \\ Fukushima, Carlo Milani
}

\section{INTRODUCTION}

The use of internal fixation for spine surgery has become a standard approach for virtually all types of procedures that require bone stabilization. The potential advantages include rigid fixation with early mobilization and more comfort in the post-operative period. However, complications like wound infections can jeopardize the final outcome of these procedures. These infections range from small areas of dehiscence to large wounds with hardware exposure. The conventional approach of serial wound debridement and antibiotic administration can last for a very long period and is only successful in cases of mild infections. Paraspinous muscle flaps have been reported to be a simple and very effective method of treating difficult wounds. ${ }^{1-3}$ However, in the more severe cases of infection with exposed hardware, the extensive skin and muscle reactive fibrosis adjacent to the wound makes the paraspinous muscle flaps difficult, if not impossible to dissect. Moreover, even if they could be dissected, they would not have sufficient volume to fill these wide and deep wounds. In this situation, the pedicled latissimus dorsi and trapezius muscle flaps have proven to be the most reliable methods of treatment. ${ }^{4-6}$ We present our experience in the management of these more complex wounds with hardware exposure in two patients, where we used combined pedicled flaps.

\section{CASE REPORTS}

Case 1. A 48 -year-old man presented with severe pain in the thoracolumbar area that did not respond to conservative therapy. During the initial investigation, an advanced multiple myeloma was diagnosed. The patient underwent chemotherapy, but the tumor grew fast, causing instability at the thoracic level and motor deficits. He was submitted

Hospital Estadual Mario Covas, Faculdade de Medicina do ABC - Santo André/SP, Brazil.

alvarocho73@hotmail.com to decompression and fusion from $\mathrm{T} 5$ to $\mathrm{T} 9$ with pedicled screw fixation. The motor deficit was significantly improved and the patient partially recovered his ability to walk. However, there was an early dehiscence of the incision with infection that was not responsive to conventional treatment with serial wound debridements, antibiotic treatment, and hyperbaric oxygen therapy. At the end of the third month of hospitalization, the wound was very wide and deep, exposing the instrumentation and the vertebral bodies (Figure1). The patient underwent a left latissimus dorsi pedicled flap with a large transverse skin paddle (Figure 2). The flap was laterally based through the thoracodorsal vessels. The muscle was used to fill the cavity while the skin paddle was primarily used to close the wound. However, in the early postoperative period, the distal extremity of the flap developed a moderate congestion with retraction and partial necrosis of the muscle and skin (Figure 3). This resulted in recurrence of the dehiscence and infection re-exposing about $20 \%$ of the instrumentation (Figure 4). Two weeks later, after proper wound debridement, the right latissimus dorsi was rotated. At this time, the skin island was drawn more proximally over the muscle to improve its vascularization. With this second

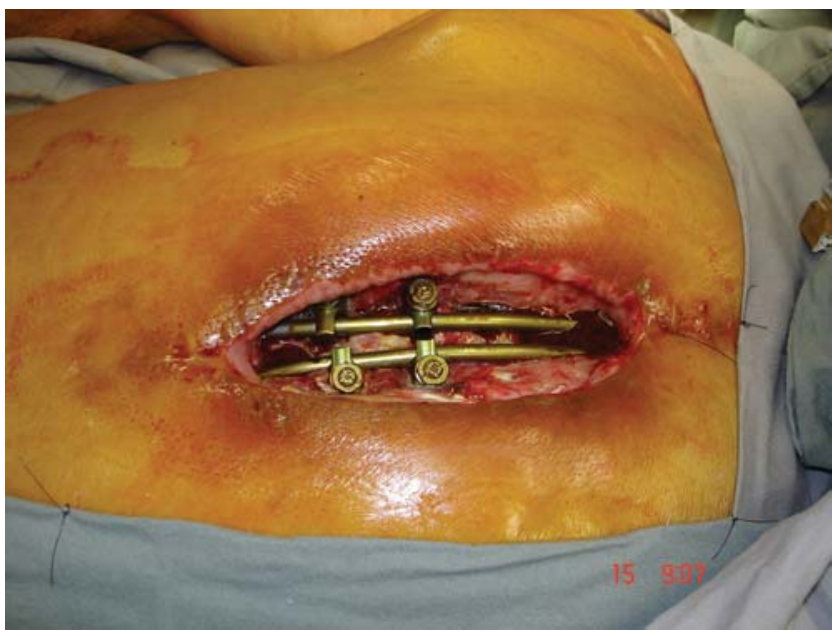

Figure 1 - Pre-operative aspect of the wound before soft tissue coverage. The instrumentation was completely exposed 


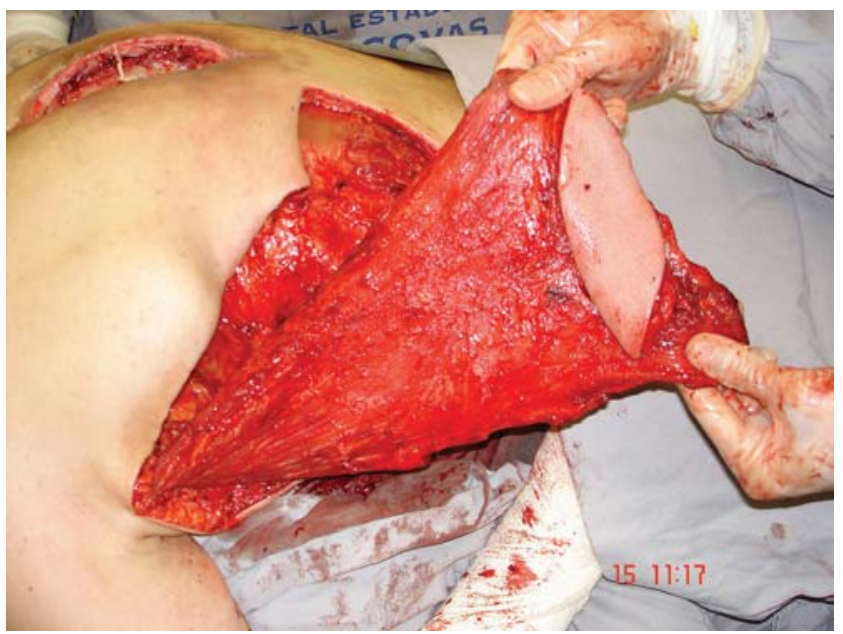

Figure 2 - The left latissimus dorsi was elevated with a transversely oriented skin paddle at the distal tip of the muscle

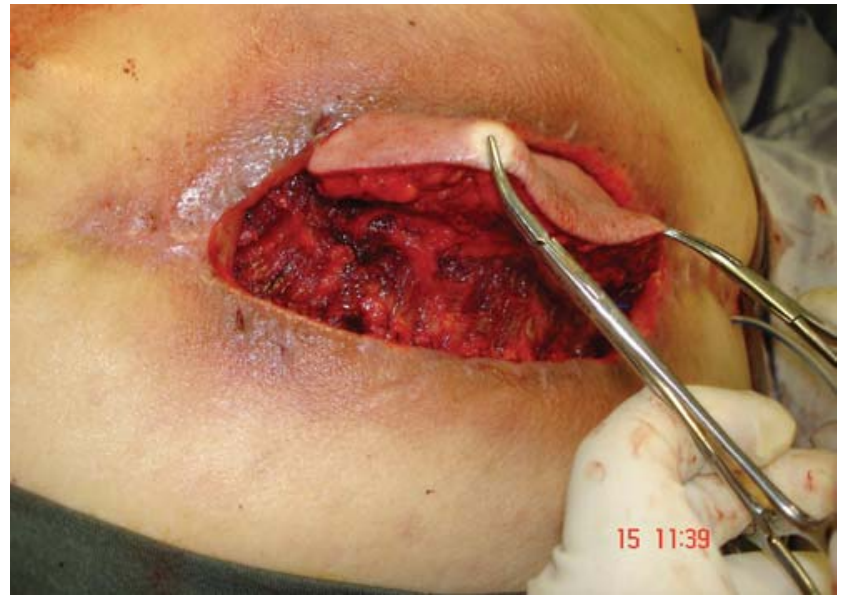

Figure 3 - The latissimus dorsi was rotated and tunelized to reach the midline of the back wound. The muscle was used to fill the defect, while the skin was used to close the wound primarily

flap, the wound was completely covered and filled, and the infection was resolved (Figure 5).

Case 2. A 9-year-old female with a myelomeningocele, presented with a severe lumbar kyphosis that hampered her ability to remain seated. In addition, the skin overlying the apex of the curvature developed sore pressures. The patient was submitted to L2 and L3 vertebral body excision to correct kyphosis and T7 to $\mathrm{S} 1$ were fused with pedicled screw fixation. At this time, the spine surgeons already encountered some difficulties with closing the incision due to attenuated skin overlying the spine. Consequently, there was an early massive dehiscence of the wound with implant exposure and infection (Figure 6). After four weeks of hyperbaric oxygen therapy, there was a significant improvement in the wound and infection; however, it was not enough to cover the implant (Figure 7). The patient underwent a left latissimus dorsi turnover flap to cover the implant exposure in the

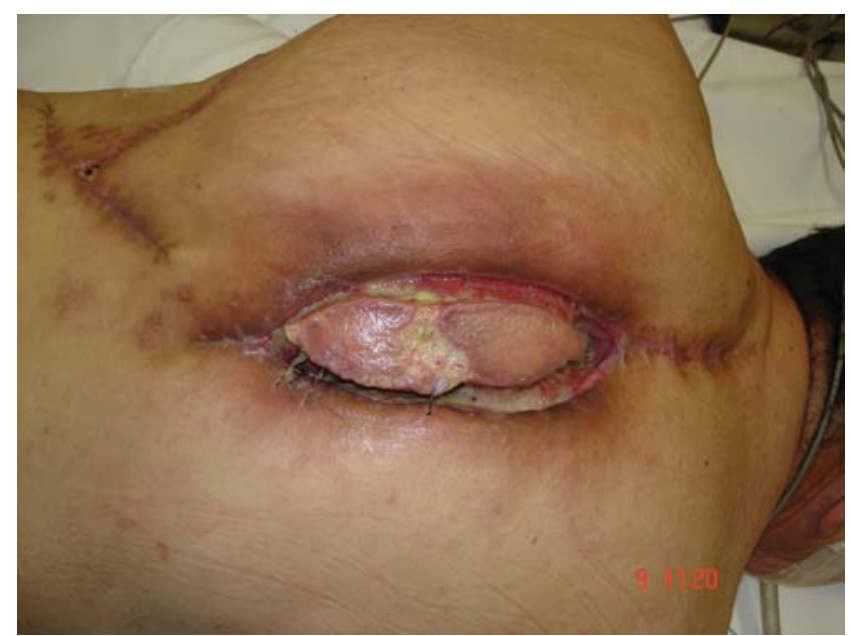

Figure 4 - Partial necrosis of the distal tip of the flap with consequent retraction and partial re-exposure of the implant

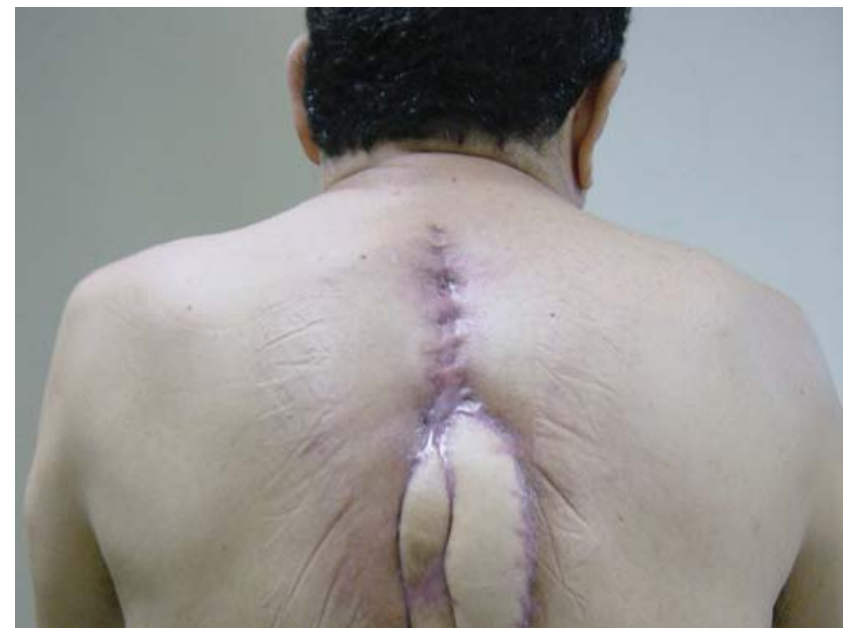

Figure 5 - Final aspect of the healed wound after rotation of the right latissimus dorsi musculocutaneous flap

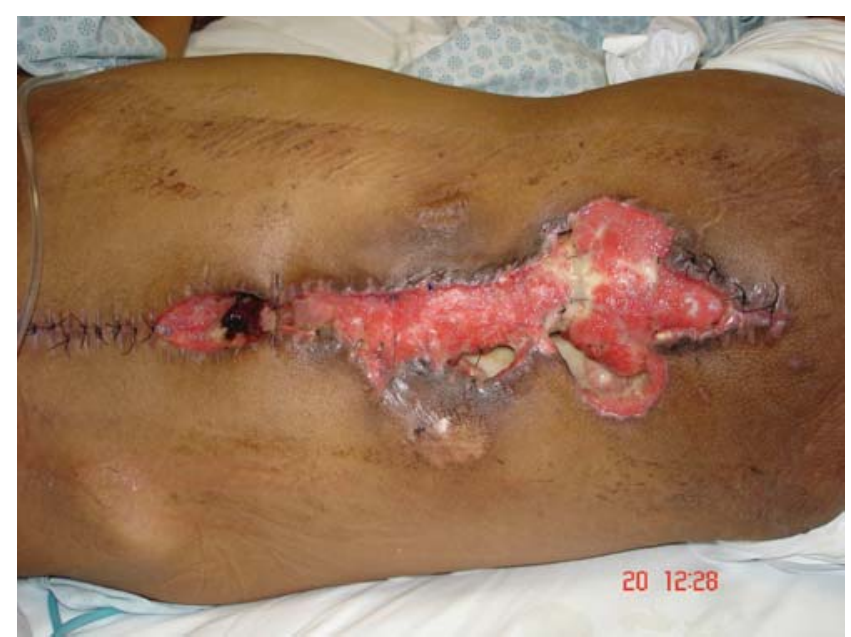

Figure 6 - Early massive dehiscence of the incision after spine surgery

lower lumbar area and a left partial trapezius turnover flap to cover a small thoracic dehiscence, simultaneously (Figure 8). 


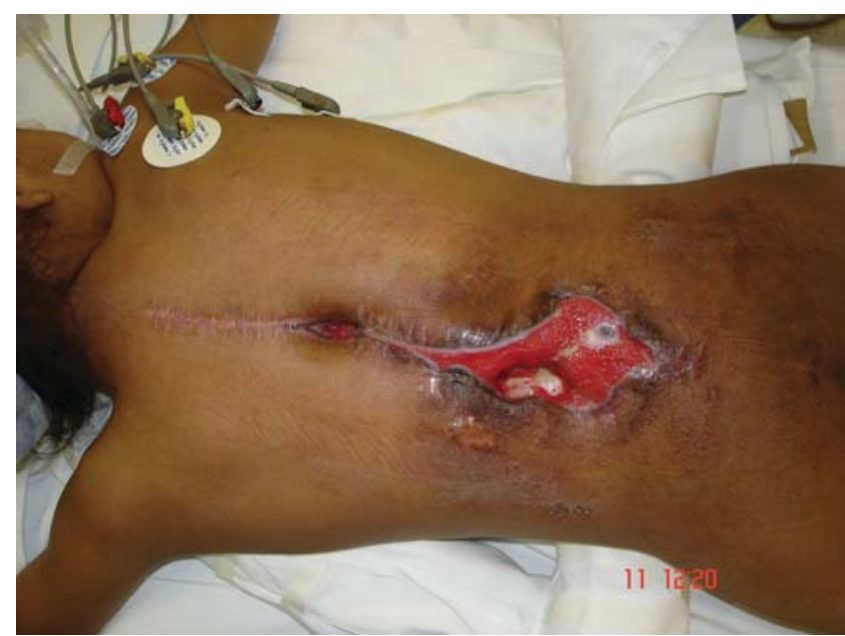

Figure 7 - After serial wound debridement and hyperbaric oxygen therapy, the infection was significantly improved, but a deep wound still persisted at the lower lumbar level, exposing the vertebral bodies and a small portion of the implant. There was also a less severe thoracic wound that did not close

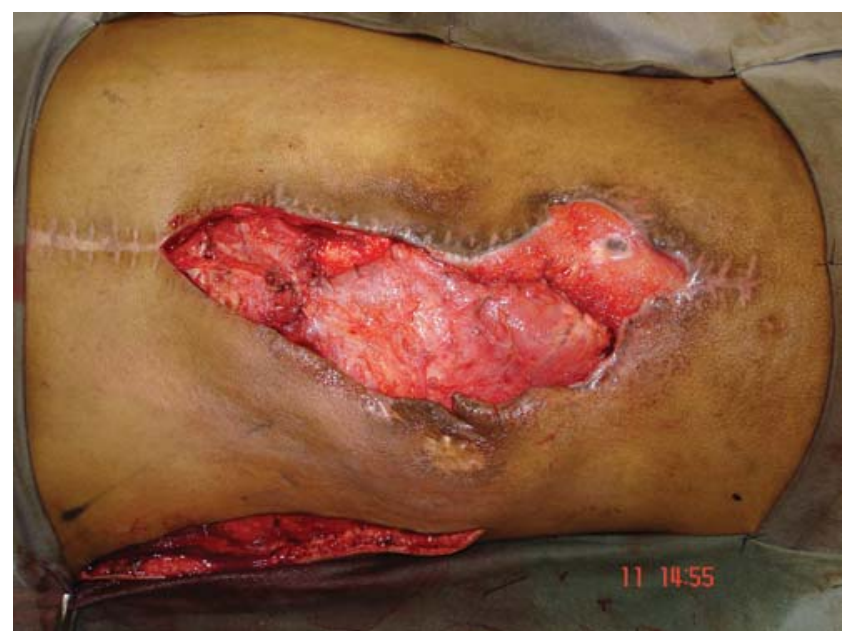

Figure 8 - The left trapezius and latissimus dorsi muscles were used as turnover flaps to cover the defects. Only the distal fifth of the trapezius was employed

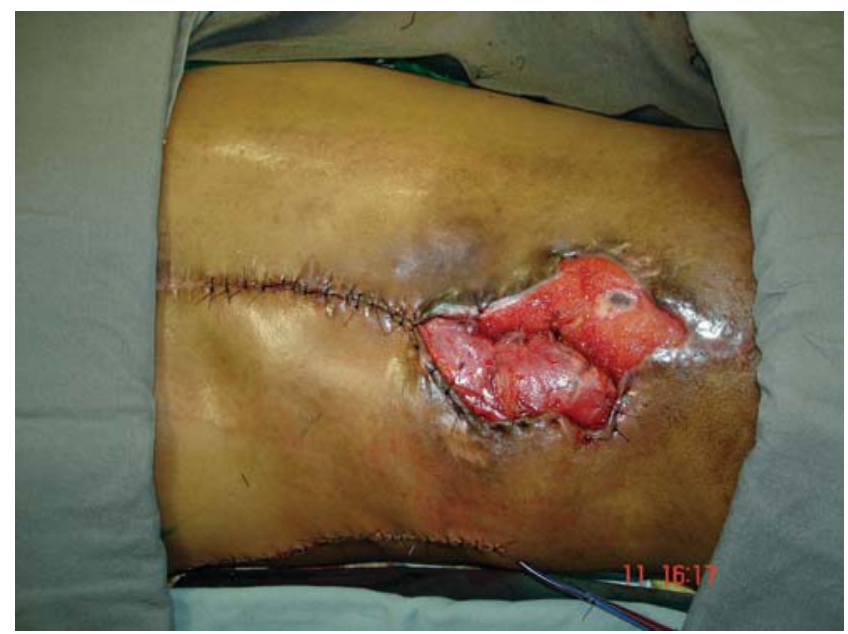

Figure 9 - The skin at the thoracic level was primarily closed over the trapezius flap

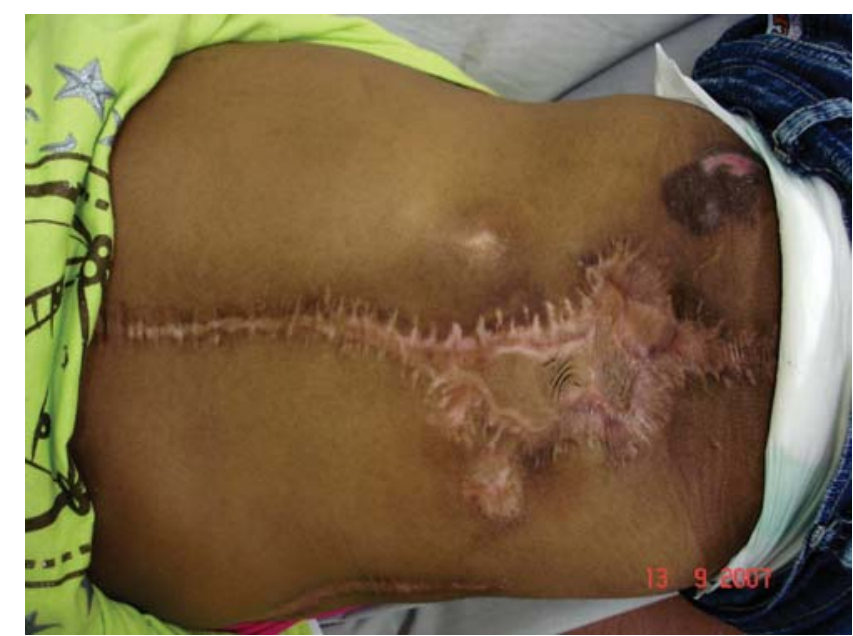

Figure 10 - The latissimus dorsi was skin grafted two weeks later. Final aspect of the healed wound

The skin in the thoracic area was closed primarily over the trapezius (Figure 9). Two weeks later, the latissimus dorsi flap was skin grafted and the patient was discharged from the hospital (Figure 10).

\section{DISCUSSION}

From May 2006 to May 2007, we performed three latissimus dorsi flaps and one partial trapezius flap in two patients. The indication for flap coverage in both was early post-operative back wound infection with exposed hardware. Two of the latissimus dorsi flaps were laterally based (thoracodorsal vessels) and placed in the same patient to cover a thoracic wound extending from T5 to T9. They were elevated in the same manner as they usually are in free flap cases, except for the skin island that was oriented transversely relative to the long axis of the flap. In the other patient, both latissimus dorsi and trapezius flaps were used as medially based (medial paraspinous perforators) turnover flaps. They were both dissected on the left side of the patient and performed simultaneously to cover a small thoracic and large lumbar defect. All flaps survived without major complications, and the spine defects were successfully covered with hardware preservation. The only minor complication occurred in the first laterally based latissimus dorsi flap that developed a partial necrosis of its distal extremity. This happened because the skin island was positioned too far distally over the tip of the muscle, where an area of poor vascularization and frequent necrosis was observed. This kind of complication was avoided in the second flap, simply by moving the skin island two fingerbreadths proximally over the muscle.

Casas and Lewis et al. reported a successful systematic regionalized approach for the reconstruction of acquired midline defects of the back using latissimus dorsi, trapezius, 
gluteus maximus, and paraspinous muscle flaps. ${ }^{5}$ Likewise, in our initial experience, the pedicled latissimus dorsi and trapezius flaps proved to be reliable tools for the treatment of severe back wound infections with exposed hardware. Klink et al. published a very concise paper describing their application in similar cases. ${ }^{4}$ They illustrated the arc of rotation of the flaps when they were used in both turnover and rotation/ advancement fashions.

Although there is frequent mention of the pedicled latissimus dorsi flap by other authors in a larger series of patients, they did not address its application as a musculocutaneous flap. ${ }^{3-6}$ We found that the possibility of taking a transverse skin island over a laterally based latissimus dorsi flap was significantly advantageous because it allowed primary closure of the back wound without necessitating a skin graft. The same was not possible when the latissimus dorsi was used as a medially based turnover flap.

Also, Dumanian et al. reported their experience with the application of free latissimus dorsi flaps for spine wounds. ${ }^{3}$
Although feasible, we were unable to observe any advantage of free flaps over the pedicled flaps in this setting.

It is important to remember that some defects located within the middle and high thoracic levels could be managed with either the trapezius or latissimus dorsi flaps. In our case, we favored the use of the latissimus dorsi flap because of the greater morbidity reported with functional loss of the trapezius. ${ }^{4}$ Nevertheless, the main reason for the preference of the latissimus dorsi flap was our previous experience with this flap in microsurgical cases.

The pedicled latissimus dorsi flap proved to be a valuable and reliable tool to treat large and deep back wound infections with hardware exposure. It can be used as a musculocutaneous advancement or rotation flap, when laterally based (thoracodorsal vessels), or as a muscular turnover flap, when medially based (paraspinous perforators). This versatility enabled the latissimus dorsi flap arc of rotation to reach midline spine defects from high thoracic through lower lumbar levels.

\section{REFERENCES}

1. Manstein ME, Manstein CH, Manstein G. Paraspinous muscle flaps. Ann. Plast. Surg. 1998;40:458-62.

2. Saint-Cyr M, Nikolis A, Moumdjian R, Frenette G, Ciaburro H, Harris $\mathrm{P}$ et al. Paraspimous muscle flaps for the treatment and prevention of cerebrospinal fluid fistulas in neurosurgery. Spine. 2003;28:E86-92.

3. Dumanian GA, Ondra SL, Liu J, Schafer MF, Chao JD. Muscle flap salvage of spine wounds with soft tissue defects or infection. Spine. 2003;28:1203-11.
4. Klink BK, Thurman T, Wittpenn GP, Lauerman WC, Cain JE. Muscle flap closure for salvage of complex back wounds. Spine. 1994;19:1467-70.

5. Casas LA, Lewis VL. A reliable approach to the closure of large acquired midline defects of the back. Plast. Reconstr. Surg. 1989;84:632-41.

6. Meiners T, Fliegar R, Jungclaus M. Use of the reverse latissimus muscle for closure of complex back wounds in patients with spine cord injury. Spine. 2003;28:1893-8 